\title{
Estudo Observacional Retrospectivo sobre o Perfil de pacientes que Receberam Terapia deSedação Paliativa em Unidade de Cuidados Paliativos de Hospital de Câncer no Brasil
}

https://doi.org/10.32635/2176-9745.RBC.2019v65n1.324

Retrospective Observational Study about Patient's Profile who Reveived Palliative Sedation Therapy at the Palliative Care Unit in a Brazilian Cancer Hospital

Estudio Observacional Retrospectivo sobre el Perfil de Pacientes que Recibieron Terapia de Sedación Paliativa en Unidad de Cuidados Paliativos de Hospital de Cáncer en Brasil

Renata Batista dos Santos'; Camila Moreira Gomes²; Carla Baioni Bonadio3; Paula de Souza Ferreira4; Raquel Barducci Bertequini5; Luís Fernando Rodrigues ${ }^{6}$

Resumo

Introdução: A Organização Mundial da Saúde estima que até 2030 ocorrerão 27 milhões de novos casos de câncer no mundo. Em 2017, esperava-se em torno de 597 mil novos casos no Brasil com letalidade de 70\%. Os cuidados paliativos incluem os cuidados de final de vida, e o controle dos sintomas físicos é um dos focos de suas açóes. Eventualmente, os sintomas podem se tornar refratários. Para esses casos, existe a opção da terapia de sedação paliativa (TSP), que é o uso de fármacos para reduzir ou abolir a consciência do paciente com o objetivo de aliviar o sofrimento. Estudos demonstram que o uso da técnica adequada do procedimento não encurta a vida do paciente. Objetivo: Identificar o perfil dos pacientes que receberam TSP no final da vida. Método: Estudo observacional retrospectivo com coleta de dados em prontuário dos pacientes falecidos na unidade de cuidados paliativos de um hospital de câncer no ano de 2015. Foram coletados dados demográficos, proporção de pacientes que necessitaram de TSP, indicações mais frequentes, drogas mais utilizadas, doses médias usadas, se discussôes com família e/ou equipe foram realizadas, duração da TSP em dias e sobrevida do paciente até o óbito. Resultados: Em 2015, ocorreram 919 óbitos; 198 (21,5\%) receberam TSP; 55,1\% do sexo feminino; média de idade de 55 anos. Os sintomas mais frequentes no momento da indicação foram dispneia (64,1\%), dor (36,3\%) e outros sintomas (30,3\%). A droga mais utilizada foi o midazolam (98\%) e a modalidade mais utilizada foi de sedaçáo contínua $(75,8 \%)$.

Palavras-chave: Sedação Profunda; Cuidados Paliativos; Neoplasias; Delírio; Estudo Observacional.

\section{Abstract}

Introduction: The World Health Organization estimates that 27 million new cases of cancer will occur until 2030 around the world. In 2017, 597.000 new cases were expected to occur in Brazil with $70 \%$ of lethality rate. Palliative Care comprises end of life care, and physical symptom control is one of the aims of its actions. Eventually, the symptoms can be refractory. For such cases, the Palliative Sedation Therapy (PST) is an option, that is the use of drugs to reduce or abolish patient's consciousness to relieve suffering. Studies have demonstrated that using suitable techniques does not shorten patient's life. Objective: To identify patient's profile that received PST at the end of life. Method: Cross-sectional observational study collecting data from patients records who died at a palliative care unit of a cancer hospital in 2015. Data was collected regarding demographics, proportion of patients who received PST, what were the more frequent symptoms, the more used drugs, the average dose used, if any discussion with the Family or the team occurred, the length of PST in days, and the survivorship until death. Results: In 2015 there were 919 deaths, 198 (21.5\%) received PST, 55.1\% were female, the average age was 55 years-old. The most frequent symptoms at the time of indication were dyspnoea $(64.1 \%)$, pain $(36.3 \%)$, and other symptoms (30.3\%). The most frequent used drug was midazolam $(98 \%)$ and the most frequent modality used was continuous sedation $(75.8 \%)$. Key words: Deep Sedation; Palliative Care; Neoplasms; Delirium; Observational Study.
Resumen

Introducción: La Organización Mundial de la Salud estima que hast 2030 deberán ocurrir 27 milhones de nuevos casos de cáncer em el mundo. En 2017, se esperaba alrededor de 597 mil nuevos casos em Brasil, com 70\% de letalidad. Los Cuidados Paliativos incluyem los cuidados de fin de vida y el control de los sintomas es uno de los focos de sus acciones. Eventualmente, los síntomas puedem se tornar refractarios. Para esos casos, existe la opción de la Terapia de Sedación Paliativa (TSP), que es el uso de fármacos para reducir ou abolir la conciencia del paciente con el objetivo de aliviar el sufrimiento. Los estudios demuestram que el uso de la técnica adecuada del procedimento no acorta la vida del paciente. Objetivo: Identificar el perfil de los pacientes que recibieron la TSP al final de la vida. Método: Estudio observacional retrospectivo con recolección de datos en prontuario de los pacientes fallecidos en la unidad de cuidados paliativos de un hospital de cáncer en el ańo 2015. Se recolectaran datos demográficos, proporción de pacientes que necesitaron TSP, indicaciones más frecuentes, drogas más utilizadas, dosis medias, se utilizaron las discusiones con la familia y / o el equipo, la duración de la TSP en días y la supervivencia del paciente hasta la muerte. Resultados: En 2015 ocurrieron 919 muertes, 198 (21,5\%) recibieron TSP, 55,1\% del sexo femenino, promedio de edad de 55 años. Los síntomas más frecuentes en el momento de la indicación fueron disnea $(64,1 \%)$, dolor $(36,3 \%)$ y otros sintomas (30,3\%). La droga más utilizada fue el midazolam $(98 \%)$ y la modalidad más utilizada fue sedación continua $(75,8 \%)$.

Palabras clave: Sedación Profunda; Cuidados Paliativos; Neoplasias; Delirio; Estudio Observacional.

${ }^{1}$ Faculdade Barretos. Barretos (SP), Brasil. Ocid iD: https://orcid.org/0000-0003-2842-7922

${ }^{2}$ Liga Acadêmica de Cuidados Paliativos da Faculdade de Ciências da Saúde de Barretos (Facisb). Barretos (SP), Brasil. Ocid iD: https://orcid.org/0000-0003-4436-0457

${ }^{3}$ Liga Acadêmica de Cuidados Paliativos da Facisb. Barretos (SP), Brasil. Ocid iD: https://orcid.org/0000-0002-9795-928X

${ }^{4}$ Liga Acadêmica de Cuidados Paliativos da Facisb. Barretos (SP), Brasil. Ocid iD: https://orcid.org/0000-0001-9740-5087

${ }^{5}$ Liga Acadêmica de Cuidados Paliativos da Facisb. Barretos (SP), Brasil. Ocid iD: https://orcid.org/0000-0002-1611-6396

${ }^{6}$ Hospital de Câncer de Barretos. Liga Acadêmica de Cuidados Paliativos da Facisb. Barretos (SP), Brasil. Ocid iD: https://orcid.org/0000-0001-6177-3769

Endereço para correspondência: Luís Fernando Rodrigues. Hospital de Câncer de Barretos. Rua Antenor Duarte Viléla, 1331 - Dr. Paulo Prata. Barretos (SP), Brasil.

CEP 14784-400. E-mail: lufe.luis@gmail.com 


\section{INTRODUÇÃO}

Segundo a Organização Mundial da Saúde, Cuidado Paliativo é uma abordagem que promove a qualidade de vida de pacientes e seus familiares, que enfrentam doenças que ameacem à continuidade da vida, por meio da prevenção e alívio do sofrimento. Requer a identificação precoce, avaliação e tratamento da dor, e outros problemas de natureza física, psicossocial e espiritual ${ }^{1}$.

O câncer é a segunda causa mundial de mortes e, em 2015, foi responsável por 8,8 milhóes de mortes no mundo. Em países de baixa e média rendas, a doença se apresenta geralmente em fase avançada ${ }^{2}$, sendo os pacientes, então, elegíveis para cuidados paliativos.

São princípios que a equipe de cuidados paliativos deve seguir: promover o alívio da dor e de outros sintomas desagradáveis; afirmar a vida e considerar a morte como um processo normal da vida; não acelerar nem adiar a morte; integrar os aspectos psicológicos e espirituais no cuidado ao paciente; oferecer um sistema de suporte que possibilite o paciente viver tão ativamente quanto o possível até o momento da sua morte; oferecer sistema de suporte para auxiliar os familiares durante a doença do paciente e a enfrentar o luto; abordagem multiprofissional para focar as necessidades dos pacientes e familiares, incluindo acompanhamento no luto; melhorar a qualidade de vida e influenciar positivamente o curso da doença; os cuidados paliativos devem ser iniciados o mais precocemente possível, juntamente com outras medidas de prolongamento da vida, como a quimioterapia e a radioterapia, e incluir todas as investigaçôes necessárias para melhor compreender e controlar situaçóes clínicas estressantes.

O Cuidado Paliativo é mais conhecido na área oncológica, porém, pode ser aplicado em outras circunstâncias de doenças sem propostas de cura ${ }^{1}$. Setenta por cento das pessoas que serão diagnosticadas com câncer no mundo irão falecer em consequência da doença que geralmente causa angústias, dor e sofrimentos nas esferas físicas, psicossociais e espirituais. Para alcançar esse objetivo, se faz necessário atender a quatro passos fundamentais ${ }^{3}$ : um bom controle de dor e outros sintomas; uma boa comunicação; apoio psicossocial; trabalho em equipe.

É comum a prevalência de sintomas perturbadores no final da vida, entre eles a dor, a dispneia, o delirium, as náuseas e os vômitos, a constipação intestinal e as secreçôes respiratórias que, se não forem bem controladas, podem levar a sofrimento intenso ${ }^{4}$. No caso de haver falha das opçóes terapêuticas, pode ser necessário recorrer à terapia de sedação paliativa (TSP) $)^{5-7}$. O termo foi proposto por Morita, Tsuneto e Shima ${ }^{8}$ após realizarem uma revisão sistematizada sobre o uso dos termos comuns ao procedimento à época, embora mais recentemente tenha-se usado o termo "terapia de sedação paliativa profunda contínua até a morte" para se referir à sedação profunda contínua? .

Ventafrida et al. ${ }^{10}$ descreveram a sedação paliativa pela primeira vez em 1990 e, desde entáo, esse conceito tem gerado discussôes e diferentes pontos de entendimento principalmente pela visão ética, uma vez que a intencionalidade de quem indica e pratica a sedação é subjetiva e poderia mascarar a intenção de abreviar a vida. Além disso, a intenção é um dos pontos que difere a TSP da eutanásia ${ }^{4}$. Sedação paliativa é definida como introdução de medicaçôes, a fim de diminuir o nível de consciência de um paciente com doença avançada ou em cuidados paliativos com a sua autorização ou de familiares, com a intenção de proporcionar alívio de sintomas refratários que não responderam à terapêutica adotada ${ }^{7}$. Sintoma refratário é definido como aquele que não pode ser adequadamente controlado a despeito de esforços intensos para identificar uma terapia que não comprometa a consciência, devendo o clínico estar alerta para o fato de as intervençôes invasivas ou não invasivas serem consideradas refratárias, sendo incapazes de promover alívio adequado, estejam associadas à morbidade aguda ou crônica intolerável e sejam improváveis de poder produzir alívio em um intervalo de tempo aceitável ${ }^{11}$. A literatura nacional é escassa de artigos e estudos nesse tema, o que justifica a realização desta pesquisa. Os objetivos foram analisar a prevalência da TSP em pacientes atendidos em uma unidade de cuidados paliativos de um hospital de câncer e como essa sedação ocorreu em termos de indicação, duração, modalidade, drogas de escolha e doses.

\section{MÉTODO}

Trata-se de um estudo observacional retrospectivo. Foram avaliados 919 prontuários de pacientes que foram a óbito em uma unidade de Cuidados Paliativos no ano de 2015. Como foram utilizados dados de todos os pacientes que foram a óbito na unidade de Cuidados Paliativos de um hospital de câncer brasileiro, esta amostra foi caracterizada como do tipo náo probabilística por conveniência. Para serem elegíveis para o estudo, os seguintes critérios de inclusão foram utilizados: pacientes que foram a óbito no ano de 2015, maiores de 18 anos de idade, de ambos os sexos e que receberam qualquer tipo de sedação. Critérios de exclusão: óbitos que não utilizaram sedaçáo, ausência de anotações sobre o assunto. Os prontuários que preencheram os critérios citados foram selecionados e as informaçóes registradas por meio de uma ficha de coleta de dados. Este trabalho seguiu a Resolução $n^{\circ}$. 466, de 12 dezembro de 2012.

Foram coletadas as seguintes informaçóes: dados demográficos (sítio primário do tumor, sítio das 
metástases, sexo, idade, escolaridade; número de pacientes que fizeram uso da TSP com relação ao número total de óbitos no ano de 2015; indicaçôes mais frequentes; drogas mais utilizadas, doses médias eficazes para produzir sedação; duração da sedação, sobrevida do paciente até o óbito; se houve reunião de equipe ou com família para decidir pelo procedimento. Este estudo foi aprovado pelo Comitê de Ética em Pesquisa (CEP) do Hospital de Câncer de Barretos sob o número 1.684/214, CAAE 57873716.2.0000.5437.

As análises descritivas das variáveis coletadas foram feitas por meio de tabelas. Para as variáveis qualitativas, foram usadas tabelas contendo os valores absolutos e relativos. Para as variáveis quantitativas, foram empregadas medidas estatísticas como a média, desvio-padrão (DP), valores mínimo, máximo e quartis.

Para avaliar a sobrevida, calculou-se o tempo decorrido desde a sedação até a morte do paciente, cujo evento de interesse foi o óbito. Nessa fase, foram utilizadas curvas de Kaplan-Meier e Testes de Log-rank.

As análises estatísticas foram feitas no software SPSS versão 21 e foi empregado o nível de significância de 5\%.

\section{RESULTADOS}

Esta amostra foi composta por 919 prontuários dos pacientes que faleceram na unidade de cuidados paliativos no ano de 2015. Desse total, 198 (21,5\%) receberam TSP, seja ela superficial ou profunda, contínua ou intermitente, portanto, considerados elegíveis para o estudo. Dos pacientes que receberam a TSP, $109(55,1 \%)$ eram do sexo feminino, tinham uma média de idade de 55 anos ao iniciar o acompanhamento com a equipe de cuidados paliativos (DP=14; 19-85), 124 (62,6\%), residiam no Estado de São Paulo e $88 \%$ tinham câncer em estádios clínicos III ou IV quando da admissão na unidade de cuidados paliativos. Os outros dados clínicos e sociodemográficos podem ser vistos na Tabela 1. Os tumores do sistema digestório foram os mais frequentemente encontrados e o perfil de diagnóstico oncológico pode ser apreciado na Figura 1.

O sintoma mais frequente no momento da indicaçáo da TSP foi a dispneia, seguida pela dor e outros sintomas. A Figura 2 traz a descriçâo de todas as indicaçóes e a Figura 3 especifica cada sintoma classificado na categoria "outros".

A modalidade de sedação contínua foi a mais frequente, sendo utilizada em 150 pacientes $(75,8 \%)$. A sedaçáo durou até o momento da morte em 188 pacientes $(95,4 \%)$, e foi suspensa antes da morte em nove pacientes. Os motivos para a suspensão foram por decisão médica em cinco pacientes, a pedido da família em dois pacientes e não foi possível encontrar o motivo registrado em dois pacientes.
Tabela 1. Dados sociodemográficos e clínicos

\begin{tabular}{|c|c|c|}
\hline & $\mathbf{N}$ & $\%$ \\
\hline Total de óbitos em 2015 & 919 & 100,0 \\
\hline Sedados & 198/919 & 21,5 \\
\hline $\begin{array}{l}\text { Terapia de sedação paliativa } \\
\text { contínua }\end{array}$ & $150 / 198$ & 75,8 \\
\hline $\begin{array}{l}\text { Terapia de sedação paliativa } \\
\text { intermitente }\end{array}$ & $48 / 198$ & 24,2 \\
\hline Permaneceu sedado até o óbito & $188 / 198$ & 95,0 \\
\hline Sexo Feminino & 109/198 & 55,0 \\
\hline $\begin{array}{l}\text { Média da idade na entrada na } \\
\text { Unidade de Cuidado Paliativo } \\
\text { (anos) }\end{array}$ & 54 & - \\
\hline
\end{tabular}

\section{Estadiamento clínico}

\begin{tabular}{l|c|c}
\hline Estádio I & 7 & 3,5 \\
\hline Estádio II & 13 & 6,5 \\
\hline Estádio III & 36 & 18,2 \\
\hline Estádio IV & 111 & 56,0 \\
\hline
\end{tabular}

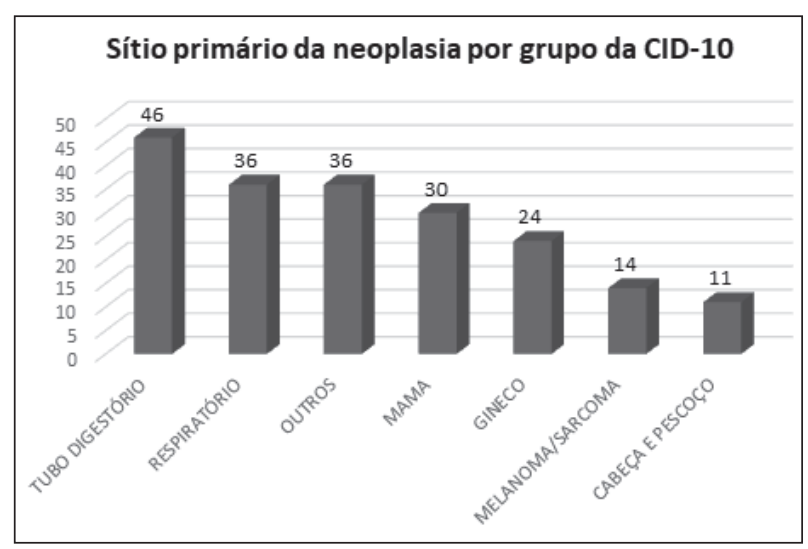

Figura 1. Distribuição dos diagnósticos mais frequentes

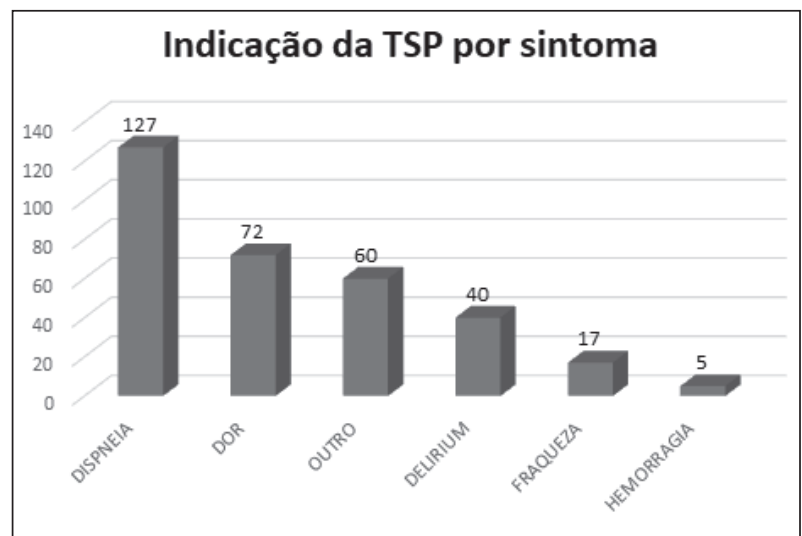

Figura 2. Distribuição das indicações mais frequentes

O midazolam foi a droga de escolha em 194 pacientes (98\%) dos casos, seguido pelo fenobarbital em três casos $(1,5 \%)$, e levomepromazina em um caso $(0,5 \%)$. A dose inicial média expressa em $\mathrm{mg} / 24$ horas de midazolam 


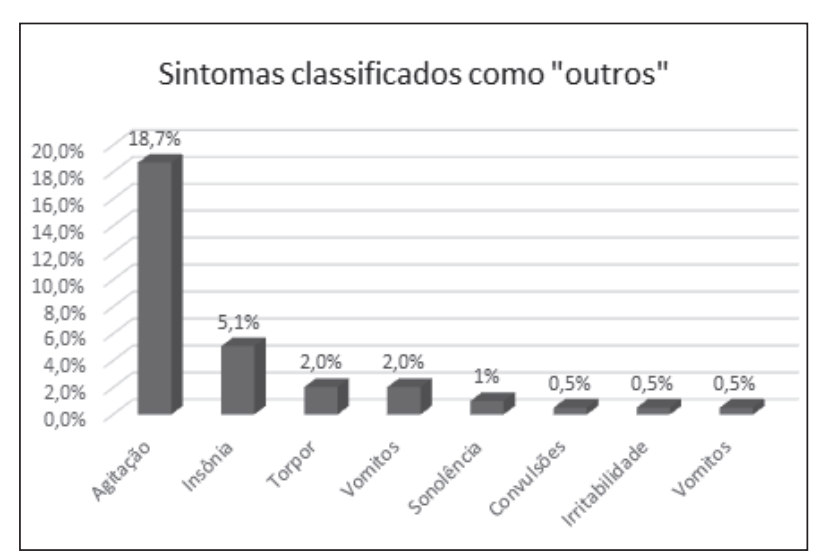

Figura 3. Sintomas classificados como "outros"

requerida foi de $23,06 \mathrm{mg}(\mathrm{DP}=22,94 ; 2,00-200,0)$ e a dose média, após a titulação em que se obteve a sedação foi de 32,41 mg ( $\mathrm{DP}=28,47 ; 2,00-185,00)$. O tempo da sedação até o óbito na modalidade contínua foi de 2,77 dias ( $\mathrm{DP}=0,36 ; 2,07-3,47)$ e na modalidade de sedação intermitente foi de 8,18 dias ( $\mathrm{DP}=1,5 ; 5,3-11,1)$.

A sobrevida dos pacientes submetidos à TSP intermitente foi maior que a dos pacientes submetidos à TSP contínua (Figura 4), sendo que a média e a mediana podem ser apreciadas na Tabela 2.

Com relação à tomada de decisão em conjunto com a família, a conversa aconteceu e foi registrada em

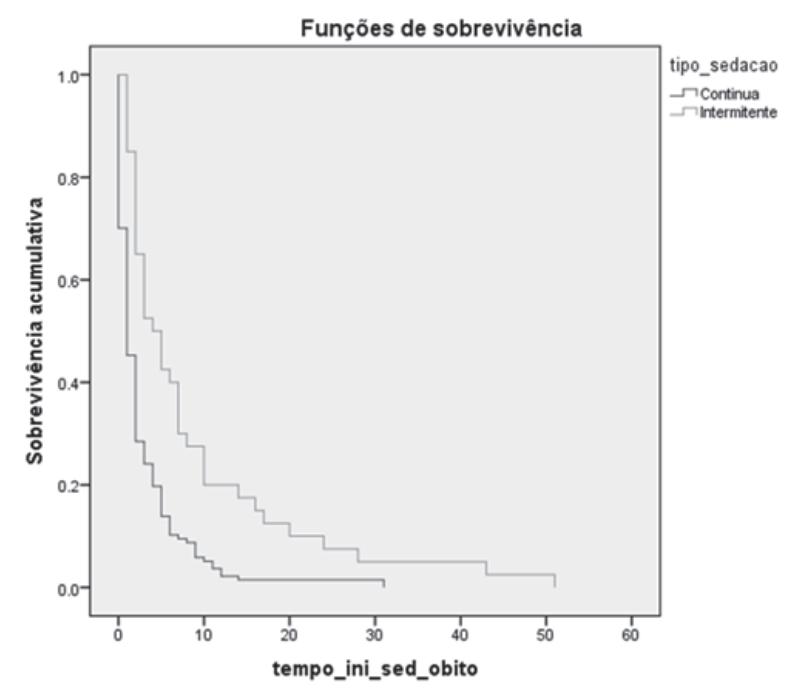

Figura 4. Comparação das curvas de sobrevida entre a sedação intermitente e a sedação contínua

Tabela 2. Sobrevida após início da sedação (dias)

\begin{tabular}{l|c|c}
\hline \multirow{2}{*}{\multicolumn{1}{c}{ Tipos de Sedação }} & \multicolumn{2}{c}{ Sobrevida } \\
\cline { 2 - 3 } & Média & Mediana \\
\hline Contínua & 2,77 & 1,00 \\
\hline Intermitente & 8,18 & 5,00 \\
\hline
\end{tabular}

prontuário 140 vezes $(71,1 \%)$ e, com a equipe, esse registro foi encontrado em 13 oportunidades $(6,6 \%)$.

\section{DISCUSSÃO}

Alguns pacientes, nas últimas semanas de vida, experimentam sofrimento considerado intolerável, no qual os procedimentos tidos como melhor prática clínica foram utilizados ao limite e para os quais a TSP passa a ser uma forte opçáo terapêutica ${ }^{5}$. Apesar de estar sendo empregada desde a década de 1990 do século $\mathrm{XX}^{10}$, é um procedimento que ainda provoca algumas controvérsias em razão da interpretação ética que se faz da TSP, pois até recentemente se acreditava que ela antecipava a morte do paciente ${ }^{12}$ e, ao se abolir a consciência do paciente, este era colocado em situação equivalente à eutanásia, chamada entáo de eutanásia lenta ${ }^{13}$. Apesar dessas controvérsias, ela continua sendo praticada largamente. Em 2015, a biblioteca Cochrane publicou uma revisão sistematizada que obteve 14 estudos válidos para a análise de acordo com os critérios de inclusão estabelecidos para essa revisão. No entanto, não houve homogeneidade suficiente para se produzir uma meta-análise e as análises de viés foram muito ruins, não sendo, portanto, possível produzir evidência de boa qualidade ${ }^{14}$. Assim é que as recomendaçóes mais recentes têm sido baseadas em consensos sobre aquilo que é mais praticado ${ }^{12,15}$.

As publicaçóes sobre o tema no território Latino Americano são raras, o que motivou a realização desta pesquisa. De acordo com dados da literatura mundial, a prevalência média da TSP é de $27 \%$, variando de $12 \%$ a $67 \%{ }^{14}$. Nossos dados mostraram uma prevalência de $21,5 \%$, próxima da média global, porém um pouco abaixo dessa média. Não há evidência de que recomendem ou padronizem um valor específico como valor de referência para a prevalência da TSP.

Neste estudo, a prática da TSP contínua foi a mais prevalente, tendo sido aplicada em 105 pacientes, correspondendo a 75,8\% dos pacientes. No entanto, não foi previsto o registro sobre se a modalidade escolhida foi superficial ou profunda, ainda que a literatura seja pródiga sobre a sedação profunda e contínua ${ }^{16-19}$ e escassa quanto à superficial e intermitente, o que gera interpretaçóes ambíguas sobre a intenção do procedimento quando da sua indicaçãa ${ }^{18}$.

Dos 14 estudos elegidos para a revisão da Cochrane, dez $(71,4 \%)$ relataram qual a indicação para se iniciar a TSP. Destes, em seis estudos, o delirium figurou como a primeira indicação, tendo aparecido em sete estudos. A dispneia, embora tenha aparecido como primeira indicação em apenas dois, esteve presente em nove estudos como uma das principais indicaçôes para a sedação. A 
agitação também foi descrita como motivo para indicar a TSP. No entanto, a agitaçáo pode ser um dos sintomas do delirium e, por isso, a frequência do delirium pode estar sendo subestimada em função da forma variada com a qual é descrito. No nosso estudo, a dispneia foi a primeira indicação em $64,1 \%$ das vezes, sendo seguida pela dor $(36,4 \%)$ das indicaçôes, e outros em terceiro lugar com $30,3 \%$ destas. Esses dados diferem dos encontrados em um estudo austríaco no qual o sintoma mais frequente foi o delirium (51\%), seguido do distress existencial $(32 \%)$ e dor $(20 \%)^{20}$. Também diferiu de outro estudo em que os motivos para sedar declarado pelo profissional especialista em cuidados paliativos foram dispneia (56\%), delirium (53.9\%) e dor (50\%), em um questionário de múltipla escolha ${ }^{16} \mathrm{e}$, também, coincidiu com um estudo alemão que encontrou como indicação agitação terminal $(56 \%)$, dispneia (39\%) e dor $(32 \%)^{21}$. Acredita-se que a forma como a informação está registrada nos documentos hospitalares, bem como a interpretaçáo dos pesquisadores responsáveis pela coleta dos dados, pode ter provocado viés no resultado no qual a opção "outros" aparece com muita frequência como indicação da TSP. Esse fato corrobora a hipótese de Morita, que afirma que a falta de consenso sobre uma estrutura padronizada para pesquisa bem como consenso sobre as definiçóes que concernem ao tema dos cuidados paliativos promovem resultados e conclusóes conflitantes nesse tema, propondo, para isso, um padrão para realizaçáo de pesquisas sobre TSP'. Comentando ainda sobre o motivo "outros", se referindo a outros sintomas: agitação $(18,7 \%)$, insônia $(5,1 \%)$, irritabilidade, sonolência e torpor $(0,5 \%$ cada um) podem ter sido manifestaçóes de delirium que eventualmente foram mal interpretadas, contribuindo para esse viés nos resultados. Esse acontecimento levanta a hipótese de que talvez o delirium tenha tido uma prevalência maior e tenha sido motivo mais frequente para a indicação da TSP na amostra pesquisada.

Com relação à dose média utilizada, para se iniciar o processo de TSP, foi de 23,06 mg e a dose média com a qual se obteve a sedação foi de $32,41 \mathrm{mg}$ em 24 horas. De acordo com a revisão da Cochrane, apenas três estudos dos 14 elegíveis registraram a dose de midazolam necessária para se obter sedação e a média foi de 37,06 mg em 24 horas. O midazolam tem sido escolhido como a droga de preferência com base em consenso e em virtude de suas propriedades farmacológicas de ter início de ação rápida e meia-vida curta, o que permite titulação rápida e superficialização do nível de consciência ${ }^{12,15}$.

De acordo com os dados neste estudo, a TSP durou em média 2,77 dias na modalidade contínua e 8,18 dias na modalidade intermitente. Como parâmetro de comparação, usaram-se os dados da revisão da Cochrane, que identificou oito estudos que trouxeram esse dado nos resultados. Procedeu-se ao cálculo simples das médias obtidas nos estudos e a duraçáo média foi de 1,86 dias. Com relação à sobrevida na mesma revisão, ela foi de 20,5 dias nos pacientes sedados contra 20,5 dos pacientes não sedados. Embora os dados não sejam uniformes e os autores da revisão não tenham calculado a média pela falta de homogeneidade entre os estudos, calculou-se essa média de acordo com os dados disponíveis. No nosso estudo, como não havia grupo controle, não se fez cálculo da sobrevida dos pacientes.

Consideram-se os dados apresentados consistentes, mas foram percebidas algumas fraquezas neste estudo que trazem riscos de vieses (bias) para os dados que devem ser levados em consideração na sua interpretação. Uma dessas fraquezas foi o episódio de não ter sido registrada a característica das sedaçóes de acordo com a profundidade, a fim de obter dados nacionais a esse respeito. Essa ocorrência pode ser explicada pela ausência do registro no prontuário de forma descritiva ou pelo uso das escalas de avaliação de sedação. Assim, não foi possível afirmar qual a prevalência da sedação superficial comparada à da profunda. É importante ressaltar esse detalhe, pois a literatura mundial é pródiga em produções sobre o tema da TSP contínua profunda ou sedação profunda contínua até a morte, que tem sido confrontada com o conceito de eutanásia naquele continente ${ }^{16-19,22}$. Outra fraqueza reside no fato de que foi necessário coletar os dados qualitativos na maneira como estavam registrados no prontuário. Isso pode ter afetado a interpretação de cada pesquisador no momento de registrar os dados na ficha de coleta, o que afetou, de maneira especial, dois aspectos analisados neste estudo: (I) as indicaçôes para se iniciar a TSP; e (II) o registro sobre a discussão com a equipe e com a família no processo de decisão em se iniciar a TSP. No que se refere às indicaçóes, estas não estavam descritas de maneira objetiva, o que limitou a coleta de dados aos sintomas presentes no momento da indicaçáo da TSP. Por fim, outra limitação deste trabalho é o fato de ter sido retrospectivo, o que limitou a verificação dos dados ao conteúdo que havia disponível nos registros dos pacientes e a forma como os conteúdos estavam registrados, o que leva à heterogeneidade da informaçáo e dificulta uma análise com maior consistência.

\section{CONCLUSÃO}

A prevalência da TSP em uma Unidade de Cuidados Paliativos de um Hospital de Câncer Brasileiro foi de $21,5 \%$. A droga mais usada foi o midazolam e a dose final média em 24 horas, para se obter uma sedação satisfatória, foi de 32,41 mg. A indicação mais frequente foi dispneia, 
dor e outros motivos. A modalidade de sedação profunda contínua foi a mais frequente e sua duração média foi de 2,77 dias. No entanto, pode ter havido viés de coleta de dados tanto na interpretaçáo como na forma de registro.

São necessários mais estudos prospectivos, nas diferentes regiôes e serviços do país, para avaliar se os parâmetros analisados neste estudo sofrem influência de algum fator da realidade local.

É necessário estimular as equipes de cuidados paliativos para que registrem de forma clara e ampla os aspectos relacionados com o processo de indicação e realização da TSP, quais sejam: a indicação (motivo) para a TSP, qual a modalidade (se contínua ou intermitente, se superficial ou profunda), a discussão com a equipe e se isso traz desconforto para algum membro da equipe, e a discussão com a família. Igualmente importante é deixar registrado qual a droga de escolha, a dose inicial e a dose que foi eficaz para se produzir o nível de sedação desejado, o tempo de sedação e a sobrevida dos pacientes.

\section{CONTRIBUIÇÕES}

Renata Batista dos Santos contribuiu substancialmente na concepção, no planejamento, na obtenção, análise e interpretação dos dados e na redação. Camila Moreira Gomes, Carla Baioni Bonadio, Paula de Souza Ferreira e Raquel Barducci Bertequini contribuíram na obtençấo dos dados. Luís Fernando Rodrigues contribuiu substancialmente na concepçáo, no planejamento, na obtenção, análise e interpretação dos dados, na redação e revisão crítica. Todos os autores aprovaram a versão final a ser publicada.

\section{DECLARAÇÃO DE CONFLITO DE INTERESSES}

Nada a declarar.

\section{FONTES DE FINANCIAMENTO}

Não há.

\section{REFERÊNCIAS}

1. World Health Organization. Palliative Care [Internet]. Geneve: WHO; 2018 Feb. 19. [cited 2018 Aug 14]. Available from: http://www.who.int/news-room/factsheets/detail/palliative-care.

2. World Health Organization. Cancer [Internet]. Geneve: WHO; 2018 Sep 12. [cited 2018 Aug 14]. Available from: http://www.who.int/news-room/fact-sheets/detail/ cancer.

3. Bruera E, De Lima L, editores. Cuidados paliativos: guías para el manejo clínico [Internet]. 2. ed. Washington,
DC: OPS/OMS; [2004?]. [cited 2018 Aug 14]. Available from: https://www.paho.org/hq/dmdocuments/2012/ PAHO-Guias-Manejo-Clinico-2002-Spa.pdf. Spanish.

4. Albert RH. End-of-Life Care: managing common symptoms. Am Fam Physician. 2017 Mar 15;95(6):356361.

5. de Graeff A, Dean M. Palliative sedation therapy in the last weeks of life: a literature review and recommendations for standards. J Palliat Med. 2007 Feb;10(1):67-85. doi: https://doi.org/10.1089/jpm.2006.0139.

6. Maltoni M, Pittureri C, Scarpi E, et al. Palliative sedation therapy does not hasten death: results from a prospective multicenter study. Ann Oncol. 2009 Jul;20(7):11631169. doi: https://doi.org/10.1093/annonc/mdp048.

7. Morita T, Tsuneto S, Shima Y. Definition of sedation for symptom relief: a systematic literature review and a proposal of operational criteria. J Pain Symptom Manage. 2002 Oct;24(4):447-453. doi: https://doi.org/10.1016/ S0885-3924(02)00499-2.

8. Morita T, Tsuneto S, Shima Y. Proposed definitions for terminal sedation. Lancet. $2001 \mathrm{Jul}$ 28;358(9278):335336. doi: https://doi.org/10.1016/S01406736(01)05515-5.

9. Morita T, Imai K, Yokomichi N, et al. Continuous Deep Sedation: a proposal for performing more rigorous empirical research. J Pain Symptom Manage. 2017 Jan;53(1):146-152. doi: https://doi.org/10.1016/j. jpainsymman.2016.08.012.

10. Ventafridda V, Ripamonti C, De Conno F, et al. Symptom prevalence and control during cancer patients' last days of life. J Palliat Care. 1990 Autumn;6(3):7-11. doi: https://doi.org/10.1177/082585979000600303.

11. Cherny NI, Portenoy RK. Sedation in the management of refractory symptoms: guidelines for evaluation and treatment. J Palliat Care. 1994 Summer;10(2):31-38. doi: https://doi.org/10.1177/082585979401000207.

12. Cherny NI, Radbruch L. The Board of the European Association for Palliative Care. European Association for Palliative Care (EAPC) recommended framework for the use of sedation in palliative care. Palliat Med. 2009 Oct;23(7):581-593. doi: https://doi. org/10.1177/0269216309107024.

13. Juth N, Lindblad A, Lynöe N, et al. European Association for Palliative Care (EAPC) framework for palliative sedation: an ethical discussion. BMC Palliat Care. 2010 Sep 13;9:20. doi: https://doi.org/10.1186/1472684X-9-20.

14. Beller EM, van Driel ML, McGregor L, et al. Palliative pharmacological sedation for terminally ill adults. Cochrane Database Syst Rev. 2015 Jan 2;1:CD010206. doi: https://doi.org/10.1002/14651858.CD010206. pub2.

15. Cherny NI; ESMO Guidelines Working Group. ESMO clinical practice guidelines for the management of 
refractory symptoms at the end of life and the use of palliative sedation. Ann Oncol. 2014 Sep;25 (Suppl 3):iii143-iii152. doi: https://doi.org/10.1093/annonc/ mdu238.

16. Ziegler S, Schmid M, Bopp M, et al. Continuous deep sedation until death in patients admitted to palliative care specialists and internists: a focus group study on conceptual understanding and administration in German-speaking Switzerland. Swiss Med Wkly. 2018 Aug 13;148:w14657. doi: https://doi.org/10.4414/ smw.2018.14657.

17. Robijn L, Cohen J, Rietjens J, et al. Trends in continuous deep sedation until death between 2007 and 2013: a repeated nationwide survey. PLoS One. 2016;11(6):e0158188. doi: https://doi.org/10.1371/ journal.pone.0158188.

18. den Hartogh G. Continuous deep sedation and homicide: an unsolved problem in law and professional morality. Med Health Care Philos. 2016 Jun;19(2):285-297. doi: https://doi.org/10.1007/s11019-015-9680-3.

19. Horn R. The 'French exception': the right to continuous deep sedation at the end of life. J Med Ethics. 2018 Mar;44(3):204-205. doi: http://dx.doi.org/10.1136/ medethics-2017-104484.

20. Schur S, Weixler D, Gabl C, et al. Sedation at the end of life - a nation-wide study in palliative care units in Austria. BMC Palliat Care. 2016 May 14;15:50. doi: https://doi.org/10.1186/s12904-016-0121-8.

21. Hopprich A, Günther LD, Laufenberg-Feldmann $\mathrm{R}$, et al. [Palliative sedation at a university palliative care unit--a descriptive analysis]. Dtsch Med Wochenschr. 2016 Apr;141(8):e60-e66. doi: https:// doi.org/10.1055/s-0041-106460

22. ten Have H, Welie JV. Palliative sedation versus euthanasia: an ethical assessment. J Pain Symptom Manage. 2014 Jan;47(1):123-136. doi: https://doi. org/10.1016/j.jpainsymman.2013.03.008. 